\title{
An elementary approach to Brownian local time based on simple, symmetric random walks
}

\author{
Tamás Szabados*†and Balázs Székely \\ Budapest University of Technology and Economics \\ Dedicated to the 70th birthdays of Endre Csáki and Pál Révész
}

\begin{abstract}
In this paper we define Brownian local time as the almost sure limit of the local times of a nested sequence of simple, symmetric random walks. The limit is jointly continuous in $(t, x)$. The rate of convergence is $n^{\frac{1}{4}}(\log n)^{\frac{3}{4}}$ that is close to the best possible. The tools we apply are almost exclusively from elementary probability theory.
\end{abstract}

\section{Introduction}

The present work is part of a bigger project that aims to rebuild stochastic calculus using almost sure (strong) approximations by simple, symmetric random walks (RW's), applying tools almost exclusively from elementary probability theory. The most advanced tool one needs for this project is a large deviation inequality. The underlying motivation is partly didactic, partly the belief that this elementary approach may help in attacking some harder problems. The prototype of such efforts was the construction of Brownian motion (BM = Wiener process) as an almost sure limit of simple RW paths, given by Frank Knight in 1962 6. This lead him to a related construction of Brownian local time via the now celebrated Ray-Knight theory in 1963 [7. An important contribution to the theory of local time was given by Pál Révész in 1981 [ in which he showed that Brownian local time can be almost surely approximated by the local time of a simple, symmetric RW with rate of convergence $n^{\frac{1}{4}+\epsilon}$. Further contributions and generalizations to this were given by Endre Csáki and Pál Révész in 1983 [3], A.N. Borodin in 1989 [2], and several others. M. Csörgő and L. Horváth in 19894 gave a best possible strong approximation based on Skorohod embedding with rate $n^{\frac{1}{4}}(\log n)^{\frac{1}{2}}(\log \log n)^{\frac{1}{4}}$. This was generalized for a wide class of RW's by R.F. Bass and D. Khoshnevisan in 1993 1.

\footnotetext{
* Corresponding author, address: Department of Mathematics, Budapest University of Technology and Economics, Müegyetem rkp. 3, H ép. V em. Budapest, 1521, Hungary, e-mail: szabados@math.bme.hu, telephone: (+36 1) 463-1111/ext. 5907, fax: (+36 1) 463-1677

${ }^{\dagger}$ Research supported by a Hungarian National Research Foundation (OTKA) grant No. T42496.

${ }^{\ddagger}$ Research supported by the HSN laboratory of BUTE.

2000 MSC. Primary 60J55, 60J65. Secondary 60F15.

Keywords and phrases. Local time, strong approximation, Brownian motion, random walk.
} 
The level sets $\{t: W(t)=x, 0 \leq t<\infty\}$ of Brownian motion have zero Lebesgue measure, hence it is not at all obvious that Brownian local time given by the classical definition of P. Lévy

$$
\mathcal{L}(t, x)=\lim _{\epsilon \searrow 0} \frac{1}{2 \epsilon} \lambda\{s \in[0, t]: W(s) \in(x-\epsilon, x+\epsilon)\},
$$

where $\lambda$ denotes Lebesgue measure, is a well-defined, non-vanishing process which has a version jointly continuous in $(t, x)$. This fact was first shown by Trotter in 1958 [13. In this paper we define Brownian local time as the almost sure limit of the local times of a nested sequence of simple, symmetric RW's. This limit is automatically jointly continuous in $(t, x)$. The rate of convergence is $n^{\frac{1}{4}}(\log n)^{\frac{3}{4}}$ that is close to the best possible.

\section{From random walks to Brownian motion}

A main tool of the present paper is an elementary construction of BM. The specific construction we are going to use in the sequel, taken from [10, is based on a nested sequence of simple, symmetric random walks that uniformly converges to the Wiener process on bounded intervals with probability 1 . This will be called "twist and shrink" construction or RW construction in the sequel.

We summarize the major steps of the "twist and shrink" construction here. We start with an infinite matrix of independent and identically distributed (i.i.d.) random variables $X_{m}(k), \mathbf{P}\left\{X_{m}(k)= \pm 1\right\}=1 / 2(m \geq 0, k \geq 1)$, defined on the same underlying probability space $(\Omega, \mathcal{F}, \mathbf{P})$. Each row of this matrix is a basis of an approximation of the Wiener process with a dyadic step size $\Delta t=2^{-2 m}$ in time and a corresponding step size $\Delta x=2^{-m}$ in space: $S_{m}(0)=0, S_{m}(n)=\sum_{k=1}^{n} X_{m}(k)(n \geq 1)$.

The second step of the construction is twisting. From the independent RW's we want to create dependent ones so that after shrinking temporal and spatial step sizes, each consecutive RW becomes a refinement of the previous one. Since the spatial unit will be halved at each consecutive row, we define stopping times by $T_{m}(0)=0$, and for $k \geq 0$,

$$
T_{m}(k+1)=\min \left\{n: n>T_{m}(k),\left|S_{m}(n)-S_{m}\left(T_{m}(k)\right)\right|=2\right\} \quad(m \geq 1)
$$

These are the random time instants when a RW visits even integers, different from the previous one. After shrinking the spatial unit by half, a suitable modification of this RW will visit the same integers in the same order as the previous RW. We operate here on each point $\omega \in \Omega$ of the sample space separately, i.e. we fix a sample path of each RW. We define twisted RWs $\tilde{S}_{m}$ recursively for $k=1,2, \ldots$ using $\tilde{S}_{m-1}$, starting with $\tilde{S}_{0}(n)=S_{0}(n)$ $(n \geq 0)$ and $S_{m}(0)=0$ for any $m \geq 0$. With each fixed $m$ we proceed for $k=0,1,2, \ldots$ successively, and for every $n$ in the corresponding bridge, $T_{m}(k)<n \leq T_{m}(k+1)$. Any bridge is flipped if its sign differs from the desired:

$$
\tilde{X}_{m}(n)=\left\{\begin{aligned}
X_{m}(n) & \text { if } S_{m}\left(T_{m}(k+1)\right)-S_{m}\left(T_{m}(k)\right)=2 \tilde{X}_{m-1}(k+1), \\
-X_{m}(n) & \text { otherwise, }
\end{aligned}\right.
$$

and then $\tilde{S}_{m}(n)=\tilde{S}_{m}(n-1)+\tilde{X}_{m}(n)$. Then $\tilde{S}_{m}(n)(n \geq 0)$ is still a simple symmetric random walk [10, Lemma 1]. The twisted RW's have the desired refinement property:

$$
\tilde{S}_{m+1}\left(T_{m+1}(k)\right)=2 \tilde{S}_{m}(k) \quad(m \geq 0, k \geq 0) .
$$

The third step of the RW construction is shrinking. The sample paths of $\tilde{S}_{m}(n)(n \geq 0)$ can be extended to continuous functions by linear interpolation, this way one gets $\tilde{S}_{m}(t)$ 
$(t \geq 0)$ for real $t$. Then we define the mth approximating $R W$ by

$$
\tilde{B}_{m}(t)=2^{-m} \tilde{S}_{m}\left(t 2^{2 m}\right) .
$$

Then the refinement property takes the form

$$
\tilde{B}_{m+1}\left(T_{m+1}(k) 2^{-2(m+1)}\right)=\tilde{B}_{m}\left(k 2^{-2 m}\right) \quad(m \geq 0, k \geq 0) .
$$

Note that a refinement takes the same dyadic values in the same order as the previous shrunken walk, but there is a time lag in general:

$$
T_{m+1}(k) 2^{-2(m+1)}-k 2^{-2 m} \neq 0 .
$$

Then we quote some important facts from [10] about the above RW construction that will be used in the sequel. These will be stated in somewhat stronger forms but can be read easily from the proofs in the cited reference, cf. Lemmas 2-4 and Theorem 3 there.

Lemma 1. Suppose that $X_{1}, X_{2}, \ldots$ is a sequence of i.i.d. random variables, $\mathbf{E}\left(X_{k}\right)=0$, $\operatorname{Var}\left(X_{k}\right)=1$, and their moment generating function is finite in a neighborhood of 0 . Let $S_{k}=X_{1}+\cdots+X_{k}, k \geq 1$. Then for any $C>1$ and $N \geq N_{0}(C)$ one has

$$
\mathbf{P}\left\{\sup _{1 \leq k \leq N}\left|S_{k}\right| \geq(2 C N \log N)^{\frac{1}{2}}\right\} \leq 2 N^{1-C} .
$$

We mention that this basic fact, that appears in the above-mentioned reference [10], essentially depends on a large deviation theorem. Also, the lemma is valid even when $N$ is not an integer.

We have a more convenient result in a special case of Hoeffding's inequality. Let $S_{1}, S_{2}, \ldots, S_{N}$ be arbitrary (finite or countable) sums of the type: $S_{k}=\sum_{r} a_{k r} X_{k r}$ with $\mathbf{P}\left\{X_{k r}= \pm 1\right\}=\frac{1}{2}, 1 \leq k \leq N$, where $X_{k r}$ and $X_{l s}$ can be dependent when $k \neq l$. Then we have the following analog of Lemma 1, cf. [11] or [12]: for any $C>1$ and $N \geq 1$,

$$
\mathbf{P}\left\{\sup _{1 \leq k \leq N}\left|S_{k}\right| \geq\left(2 C \log N \sup _{1 \leq k \leq N} \operatorname{Var}\left(S_{k}\right)\right)^{\frac{1}{2}}\right\} \leq 2 N^{1-C} .
$$

Lemma 1 easily implies that the time lags (3) are uniformly small if $m$ or $K$ are large enough.

Lemma 2. For any $C>1$, and for any $K>0$ and $m \geq 1$ such that $K 2^{2 m} \geq N_{1}(C)$, we have

$$
\begin{array}{r}
\mathbf{P}\left\{\sup _{0 \leq k 2^{-2 m} \leq K}\left|T_{m+1}(k) 2^{-2(m+1)}-k 2^{-2 m}\right| \geq\left(\frac{3}{2} C K \log { }_{*} K\right)^{\frac{1}{2}} m^{\frac{1}{2}} 2^{-m}\right\} \\
\leq 2\left(K 2^{2 m}\right)^{1-C},
\end{array}
$$

where $\log _{*} x=\max \{1, \log x\}$.

This lemma and the refinement property (2) implies the uniform closeness of two approximations of BM if $m$ or $K$ are large enough. We give a proof of the next lemma since its statement is somewhat stronger than the one of the corresponding Lemma 4 in 10]; also, we want to emphasize the similarities between this and lemmas about the local time below. 
Lemma 3. For any $C>1$, and for any $K>0$ and $m \geq 1$ such that $K 2^{2 m} \geq N_{2}(C)$, we have

(a)

$$
\begin{array}{r}
\mathbf{P}\left\{\sup _{0 \leq k 2^{-2 m} \leq K}\left|\tilde{B}_{m+1}\left(k 2^{-2 m}\right)-\tilde{B}_{m}\left(k 2^{-2 m}\right)\right| \geq \frac{11}{4} C K^{\frac{1}{4}}\left(\log _{*} K\right)^{\frac{3}{4}} m^{\frac{3}{4}} 2^{-\frac{m}{2}}\right\} \\
\leq 6\left(K 2^{2 m}\right)^{1-C}
\end{array}
$$

(b)

$$
\begin{array}{r}
\mathbf{P}\left\{\sup _{j \geq 1} \sup _{0 \leq t \leq K}\left|\tilde{B}_{m+j}(t)-\tilde{B}_{m}(t)\right| \geq 27 C K_{*}^{\frac{1}{4}}\left(\log _{*} K\right)^{\frac{3}{4}} m^{\frac{3}{4}} 2^{-\frac{m}{2}}\right\} \\
\leq \frac{6}{1-4^{1-C}}\left(K 2^{2 m}\right)^{1-C}
\end{array}
$$

where $K_{*}=\max \{1, K\}$.

Proof. (a) Using the abbreviation $t_{k}=k 2^{-2 m}$, we have

$$
\begin{aligned}
\tilde{B}_{m+1}\left(t_{k}\right)-\tilde{B}_{m}\left(t_{k}\right) & =\tilde{B}_{m+1}\left(4 k 2^{-2(m+1)}\right)-\tilde{B}_{m+1}\left(T_{m+1}(k) 2^{-2(m+1)}\right) \\
& =2^{-m-1}\left(\tilde{S}_{m+1}(4 k)-\tilde{S}_{m+1}\left(T_{m+1}(k)\right)\right)
\end{aligned}
$$

Let $D_{K, m}=C K^{\frac{1}{4}}\left(\log _{*} K\right)^{\frac{3}{4}} m^{\frac{3}{4}} 2^{-\frac{m}{2}}$. Then

$$
\begin{aligned}
& \mathbf{P}\left\{\sup _{0 \leq t_{k} \leq K}\left|\tilde{B}_{m+1}\left(t_{k}\right)-\tilde{B}_{m}\left(t_{k}\right)\right| \geq \frac{11}{4} D_{K, m}\right\} \\
& \quad \leq \mathbf{P}\left\{A_{K, m}\right\}+\sum_{k=1}^{K 2^{2 m}} \mathbf{P}\left\{\sup _{\left\{j:|j-4 k| \leq N^{\prime}\right\}}\left|\tilde{S}_{m+1}(j)-\tilde{S}_{m+1}(4 k)\right| \geq \frac{11}{2} 2^{m} D_{K, m}\right\},
\end{aligned}
$$

where

$$
A_{K, m}=\left\{\sup _{0 \leq k 2^{-2 m} \leq K}\left|T_{m+1}(k)-4 k\right| \geq N^{\prime}\right\}, \quad N^{\prime}=\left\lfloor\left(24 C m K \log _{*} K\right)^{\frac{1}{2}} 2^{m}\right\rfloor .
$$

Here we can apply Lemma 2 to the first term and inequality (4) with $N^{\prime}$ and $C^{\prime}$ to the terms in the summation above. The constant $C^{\prime}>1$ is chosen so that the two terms be of the same order: $\left(N^{\prime}\right)^{1-C^{\prime}} \approx N^{-C}$, where $N=K 2^{2 m}$. Then $\left(N^{\prime}\right)^{1-C^{\prime}} \leq\left(K^{\frac{1}{2}} 2^{m}\right)^{1-C^{\prime}}=$ $\left(K 2^{2 m}\right)^{-C}$ implies $C^{\prime}=2 C+1<3 C$. Since $\log N^{\prime} \leq m \log _{*} C \log _{*} K$ if $K$ or $m$ are large enough and

$$
\sup _{\left\{j:|j-4 k| \leq N^{\prime}\right\}} \operatorname{Var}\left(\tilde{S}_{m+1}(j)-\tilde{S}_{m+1}(4 k)\right)=N^{\prime},
$$

it follows that $\left(2 C^{\prime} N^{\prime} \log N^{\prime}\right)^{\frac{1}{2}} \leq \frac{11}{2} 2^{m} D_{K, m}$.

As a result, we obtain that

$$
\begin{aligned}
\mathbf{P} & \left\{\sup _{0 \leq t_{k} \leq K}\left|\tilde{B}_{m+1}\left(t_{k}\right)-\tilde{B}_{m}\left(t_{k}\right)\right| \geq \frac{11}{4} D_{K, m}\right\} \\
& \leq 2\left(K 2^{2 m}\right)^{1-C}+2 K 2^{2 m} 2\left(N^{\prime}\right)^{1-C^{\prime}} \leq 6\left(K 2^{2 m}\right)^{1-C} .
\end{aligned}
$$


This proves (a).

(b) Let $D_{K, m}^{*}=C K_{*}^{\frac{1}{4}}\left(\log _{*} K\right)^{\frac{3}{4}} m^{\frac{3}{4}} 2^{-\frac{m}{2}}$. By (a),

$$
\sup _{0 \leq t_{k} \leq K}\left|\tilde{B}_{m+1}\left(t_{k}\right)-\tilde{B}_{m}\left(t_{k}\right)\right|<\frac{11}{4} D_{K, m}^{*},
$$

except for an event of probability not exceeding $6\left(K 2^{2 m}\right)^{1-C}$. Consider an interval $\left[t_{k}, t_{k+1}\right]$. Clearly, $\left|\tilde{B}_{m}\left(t_{k+1}\right)-\tilde{B}_{m}\left(t_{k}\right)\right|=2^{-m} \leq 2^{-\frac{1}{2}} D_{K, m}^{*}$. On the other hand, $\tilde{B}_{m+1}$ makes 4 steps of magnitude $2^{-m-1}$ on this interval. Then the maximum deviation between $\tilde{B}_{m+1}$ and $\tilde{B}_{m}$ at the instant $t_{k+\frac{1}{4}}$ (or at $t_{k+\frac{3}{4}}$ ) cannot exceed $\frac{11}{4} D_{K, m}^{*}+\frac{1}{4} 2^{-m}+2^{-m-1} \leq\left(\frac{11}{4}+\frac{3}{4} 2^{-\frac{1}{2}}\right) D_{K, m}^{*}$. At time $t_{k+\frac{1}{2}}$ the deviation cannot be larger than $\frac{11}{4} D_{K, m}^{*}+2^{-m-1}$, which is smaller than the previous bound. Hence

$$
\mathbf{P}\left\{\sup _{0 \leq t \leq K}\left|\tilde{B}_{m+1}(t)-\tilde{B}_{m}(t)\right| \geq \frac{11+3 \cdot 2^{-\frac{1}{2}}}{4} D_{K, m}^{*}\right\} \leq 6\left(K 2^{2 m}\right)^{1-C} .
$$

Using the fact that

$$
\sum_{j=0}^{\infty}(m+j)^{\frac{3}{4}} 2^{-\frac{m+j}{2}} \leq m^{\frac{3}{4}} 2^{-\frac{m}{2}} \sum_{j=0}^{\infty}(1+j)^{\frac{3}{4}} 2^{-\frac{j}{2}}<\frac{65}{8} m^{\frac{3}{4}} 2^{-\frac{m}{2}}
$$

(the last inequality can be checked for example by MAPLE), we obtain that

$$
\begin{aligned}
& \mathbf{P}\left\{\sup _{j \geq 1} \sup _{0 \leq t \leq K}\left|B_{m+j}(t)-B_{m}(t)\right| \geq \frac{65}{8} \cdot \frac{11+3 \cdot 2^{-\frac{1}{2}}}{4} D_{K, m}^{*}\right\} \\
& \leq \sum_{j=0}^{\infty} \mathbf{P}\left\{\sup _{0 \leq t \leq K}\left|B_{m+j+1}(t)-B_{m+j}(t)\right| \geq \frac{11+3 \cdot 2^{-\frac{1}{2}}}{4} D_{K, m+j}^{*}\right\} \\
& \leq \sum_{j=0}^{\infty} 6\left(K 2^{2(m+j)}\right)^{1-C}=\frac{6}{1-4^{1-C}}\left(K 2^{2 m}\right)^{1-C} .
\end{aligned}
$$

Since $\frac{65}{8} \cdot \frac{11+3 \cdot 2^{-\frac{1}{2}}}{4}<27$, this proves $(\mathrm{b})$.

It follows then that the above procedure gives a rather natural and nearly optimal (as far as Skorohod embedding is concerned, see below) construction of BM.

Theorem 1. On any bounded interval the sequence $\left(\tilde{B}_{m}\right)$ almost surely uniformly converges as $m \rightarrow \infty$ and the limit process is Brownian motion $W$. For any $C>1$, and for any $K>0$ and $m \geq 1$ such that $K 2^{2 m} \geq N_{2}(C)$, we have

$$
\mathbf{P}\left\{\sup _{0 \leq t \leq K}\left|W(t)-\tilde{B}_{m}(t)\right| \geq 27 C K_{*}^{\frac{1}{4}}\left(\log _{*} K\right)^{\frac{3}{4}} m^{\frac{3}{4}} 2^{-\frac{m}{2}}\right\} \leq \frac{6}{1-4^{1-C}}\left(K 2^{2 m}\right)^{1-C} .
$$

Now using the Borel-Cantelli lemma we get that for any fixed $K>0$ there is a constant $c_{K}=28 K_{*}^{\frac{1}{4}}\left(\log _{*} K\right)^{\frac{3}{4}}$ (taking $C=1+\frac{1}{27}$, say) such that almost surely,

$$
\limsup _{m \rightarrow \infty} m^{-\frac{3}{4} 2^{\frac{m}{2}}} \sup _{0 \leq t \leq K}\left|W(t)-\tilde{B}_{m}(t)\right|<c_{K} .
$$


Similarly, for any fixed $m \geq 1$ there is a constant $c_{m}=55 m^{\frac{3}{4}} 2^{-\frac{m}{2}}$ such that almost surely,

$$
\limsup _{K \rightarrow \infty} K^{-\frac{1}{4}}(\log K)^{-\frac{3}{4}} \sup _{0 \leq t \leq K}\left|W(t)-\tilde{B}_{m}(t)\right|<c_{m} .
$$

For, since $K^{\frac{1}{4}}(\log K)^{\frac{3}{4}}$ and also for any $\omega \in \Omega$, $\sup _{0 \leq t \leq K}\left|W(t)-\tilde{B}_{m}(t)\right|$ are non-decreasing in $K$, it is enough to show that

$$
\limsup _{n \rightarrow \infty} n^{-\frac{1}{4}}(\log n)^{-\frac{3}{4}} \sup _{0 \leq t \leq n+1}\left|W(t)-\tilde{B}_{m}(t)\right|<c_{m},
$$

when $n$ runs through integer values only. Since

$$
\frac{(n+1)^{\frac{1}{4}}(\log (n+1))^{\frac{3}{4}}}{n^{\frac{1}{4}}(\log n)^{\frac{3}{4}}} \leq 1+\epsilon
$$

for any $\epsilon>0$ if $n$ is large enough, taking, say, $C=2+\frac{1}{27}$ in Theorem 1 , the Borel-Cantelli lemma shows (8).

Next we are going to discuss the properties of another nested sequence of random walks, obtained by Skorohod embedding. This sequence is not identical, though asymptotically equivalent to the above RW construction, cf. [10, Theorem 4]. Given a Wiener process $W$, first we define the stopping times which yield the Skorohod embedded process $B_{m}\left(k 2^{-2 m}\right)$ into $W$. For every $m \geq 0$ let $s_{m}(0)=0$ and

$$
s_{m}(k+1)=\inf \left\{s: s>s_{m}(k),\left|W(s)-W\left(s_{m}(k)\right)\right|=2^{-m}\right\} \quad(k \geq 0) .
$$

With these stopping times the embedded process by definition is

$$
B_{m}\left(k 2^{-2 m}\right)=W\left(s_{m}(k)\right) \quad(m \geq 0, k \geq 0) .
$$

This definition of $B_{m}$ can be extended to any real $t \geq 0$ by pathwise linear interpolation. The next lemma describes some useful facts about the relationship between $\tilde{B}_{m}$ and $B_{m}$. These follow from [10, Lemmas 5,7 and Theorem 4], with some minor modifications.

In general, roughly saying, $\tilde{B}_{m}$ is more useful when someone wants to generate stochastic processes from scratch, while $B_{m}$ is more advantageous when someone needs a discrete approximation of given processes, like in the case of stochastic integration.

Lemma 4. For any $C>1$, and for any $K>0$ and $m \geq 1$ such that $K 2^{2 m} \geq N_{3}(C)$ take the following subset of the sample space:

$$
A_{K, m}^{*}=\left\{\sup _{n>m} \sup _{0 \leq k 2^{-2 m} \leq K}\left|2^{-2 n} T_{m, n}(k)-k 2^{-2 m}\right|<\left(42 C K \log _{*} K\right)^{\frac{1}{2}} m^{\frac{1}{2}} 2^{-m}\right\},
$$

where $T_{m, n}(k)=T_{n} \circ T_{n-1} \circ \cdots \circ T_{m}(k)$ for $n>m \geq 0$ and $k \geq 0$. Then

$$
\mathbf{P}\left\{\left(A_{K, m}^{*}\right)^{c}\right\} \leq \frac{2}{1-4^{1-C}}\left(K 2^{2 m}\right)^{1-C} .
$$

Moreover, $\lim _{n \rightarrow \infty} 2^{-2 n} T_{m, n}(k)=t_{m}(k)$ exists almost surely and on $A_{K, m}^{*}$ we have

$$
\tilde{B}_{m}\left(k 2^{-2 m}\right)=W\left(t_{m}(k)\right) \quad\left(0 \leq k 2^{-2 m} \leq K\right),
$$

cf. (10). Further, on $A_{K, m}^{*}$ except for a zero probability subset, $s_{m}(k)=t_{m}(k)$ and

$$
\sup _{0 \leq k 2^{-2 m} \leq K}\left|s_{m}(k)-k 2^{-2 m}\right| \leq\left(42 C K \log _{*} K\right)^{\frac{1}{2}} m^{\frac{1}{2}} 2^{-m} .
$$


If the Wiener process is built by the RW construction described above using a sequence $\left(\tilde{B}_{m}\right)$ of nested RW's and then one constructs the Skorohod embedded RW's $\left(B_{m}\right)$, it is natural to ask about rate of convergence of the latter. The answer described by the next theorem is that it is essentially the same as the one of $\left(\tilde{B}_{m}\right)$, cf. Theorem 1 ,

Theorem 2. For any $C>1$, and for any $K>0$ and $m \geq 1$ such that $K 2^{2 m} \geq \max \left\{N_{2}(C)\right.$, $\left.N_{3}(C)\right\}$ we have

$$
\mathbf{P}\left\{\sup _{0 \leq t \leq K}\left|W(t)-B_{m}(t)\right| \geq 27 C K_{*}^{\frac{1}{4}}\left(\log _{*} K\right)^{\frac{3}{4}} m^{\frac{3}{4}} 2^{-\frac{m}{2}}\right\} \leq \frac{8}{1-4^{1-C}}\left(K 2^{2 m}\right)^{1-C} .
$$

This theorem and its proof are slight modifications of Theorem 1 in [12. Kiefer [5] showed that the best possible rate of convergence one can get with Skorohod embedding is $n^{\frac{1}{4}}(\log n)^{\frac{1}{2}}(\log \log n)^{\frac{1}{4}}$. Our rate of convergence $n^{\frac{1}{4}}(\log n)^{\frac{3}{4}}, n=K 2^{2 m}$, is close to this.

\section{An elementary definition of Brownian local time}

We define the local time of the random walk $\left(\tilde{S}_{m}(k)\right)_{k=0}^{\infty}$ at a point $x \in \mathbf{Z}$ at time $k \in \mathbf{N}_{0}=$ $\{0,1,2, \ldots\}$ as $\ell_{m}(0, x)=0$ and

$$
\ell_{m}(k, x)=\#\left\{j: 0 \leq j<k, \tilde{S}_{m}(j)=x\right\} \quad(k \geq 1)
$$

This is somewhat different from the more usual definition $\tilde{\ell}_{m}(k, x)=\#\{j: 0<j \leq$ $\left.k, \tilde{S}_{m}(j)=x\right\}$, but the former fits better the construction of Brownian motion discussed in this paper. The local time of the $m$ th approximation $\tilde{B}_{m}$ at a point $x \in 2^{-m} \mathbf{Z}$ at time $t \in 2^{-2 m} \mathbf{N}_{0}$ is defined as $\mathcal{L}_{m}(t, x)=2^{-m} \ell_{m}\left(t 2^{2 m}, x 2^{m}\right)$, corresponding to the fact that the spatial step size of $\tilde{B}_{m}$ is $2^{-m}$. This is in complete agreement with (1) replacing $W$ by $\tilde{B}_{m}$ there.

Finally, we define $\mathcal{L}_{m}(t, x)$ for arbitrary $t \in \mathbb{R}_{+}$and $x \in \mathbb{R}$ by linear interpolation, making it into a continuous process:

$$
\begin{aligned}
\mathcal{L}_{m}(t, x)=\mathcal{L}_{m}\left(t_{k}, x_{j}\right) & +\frac{t-t_{k}}{t_{k+1}-t_{k}}\left(\mathcal{L}_{m}\left(t_{k+1}, x_{j+1}\right)-\mathcal{L}_{m}\left(t_{k}, x_{j+1}\right)\right) \\
& +\frac{x-x_{j}}{x_{j+1}-x_{j}}\left(\mathcal{L}_{m}\left(t_{k}, x_{j+1}\right)-\mathcal{L}_{m}\left(t_{k}, x_{j}\right)\right)
\end{aligned}
$$

if $x \geq x_{j}+2^{m}\left(t-t_{k}\right)$,

$$
\begin{aligned}
\mathcal{L}_{m}(t, x)=\mathcal{L}_{m}\left(t_{k}, x_{j}\right) & +\frac{t-t_{k}}{t_{k+1}-t_{k}}\left(\mathcal{L}_{m}\left(t_{k+1}, x_{j}\right)-\mathcal{L}_{m}\left(t_{k}, x_{j}\right)\right) \\
& +\frac{x-x_{j}}{x_{j+1}-x_{j}}\left(\mathcal{L}_{m}\left(t_{k+1}, x_{j+1}\right)-\mathcal{L}_{m}\left(t_{k+1}, x_{j}\right)\right)
\end{aligned}
$$

if $x<x_{j}+2^{m}\left(t-t_{k}\right)$, where $t_{k}=k 2^{-2 m}\left(k \in \mathbf{N}_{0}\right), x_{j}=j 2^{-m}(j \in \mathbf{Z})$, and $(t, x) \in$ $\left[t_{k}, t_{k+1}\right] \times\left[x_{j}, x_{j+1}\right]$. Our aim is to define the local time $\mathcal{L}(t, x)$ of Brownian motion as the limit of $\mathcal{L}_{m}(t, x)$ as $m \rightarrow \infty$ and to show that this limit is jointly continuous in $(t, x)$.

The local times at $x=0$ are simply denoted by $\ell_{m}(k), \tilde{\ell}_{m}(k)$, and $\mathcal{L}_{m}(t)$, respectively. Then $\ell_{m}(k)=1+\tilde{\ell}_{m}(k-1)$ for $k \geq 1$ integer. 
One can define "one-sided", up and down local times $\ell_{m}^{+}(k, x)$ and $\ell_{m}^{-}(k, x)\left(k \in \mathbf{N}_{0}\right.$, $x \in \mathbf{Z})$ as well: $\ell_{m}^{ \pm}(0, x)=0$ and

$$
\ell_{m}^{ \pm}(k, x)=\#\left\{j: 0 \leq j<k, \tilde{S}_{m}(j)=x, \tilde{S}_{m}(j+1)=x \pm 1\right\} \quad(k \geq 1) .
$$

Then $\ell_{m}(k, x)=\ell_{m}^{+}(k, x)+\ell_{m}^{-}(k, x)$. The definitions of $\mathcal{L}_{m}^{+}(t, x)$ and $\mathcal{L}_{m}^{-}(t, x)$ for $t \in \mathbb{R}_{+}$ and $x \in \mathbb{R}$ can go in the same way as the definition of $\mathcal{L}_{m}(t, x)$ above.

It can be useful to introduce an even finer division of local time, introducing up-crossing local time by

$$
\ell_{m}^{++}(k, x)=\#\left\{j: 1 \leq j<k, \tilde{S}_{m}(j-1)=x-1, \tilde{S}_{m}(j)=x, \tilde{S}_{m}(j+1)=x+1\right\}
$$

and up-bouncing local time by

$$
\ell_{m}^{-+}(k, x)=\#\left\{j: 1 \leq j<k, \tilde{S}_{m}(j-1)=x+1, \tilde{S}_{m}(j)=x, \tilde{S}_{m}(j+1)=x+1\right\},
$$

where $k \geq 2, x \in \mathbb{Z}$. Then $\ell_{m}^{+}(k, x)=\ell_{m}^{++}(k, x)+\ell_{m}^{-+}(k, x)$. The definitions of down-crossing and down-bouncing local times $\ell_{m}^{--}(k, x)$ and $\ell_{m}^{+-}(k, x)$ are similar.

As it is well-known, see e.g. [9, p. 95], the exact distribution of the local time $\tilde{\ell}$ of a simple, symmetric RW $(S(k))_{k=0}^{\infty}, S(0)=0$, is

$$
\mathbf{P}\{\tilde{\ell}(2 k)=j\}=\mathbf{P}\{\tilde{\ell}(2 k+1)=j\}=2^{-2 k+j}\left(\begin{array}{c}
2 k-j \\
k
\end{array}\right) \quad(j=0, \ldots, k) .
$$

Hence the usual argument for the De Moivre-Laplace limit theorem gives that uniformly for any $0 \leq j \leq K_{k}=o\left(k^{\frac{2}{3}}\right)$ one has

$$
\mathbf{P}\{\tilde{\ell}(k)=j\} \sim \sqrt{\frac{2}{\pi k}} \exp \left(-\frac{j^{2}}{2 k}\right) \quad(k \rightarrow \infty),
$$

where $\sim$ denotes "asymptotically equal". Then for any sequence $u_{k} \rightarrow \infty, u_{k}=o\left(k^{\frac{1}{6}}\right)$ we obtain the following large deviation inequality for local times $\ell(k, x), \ell^{+}(k, x)$, and $\ell^{-}(k, x)$ $(x \in \mathbb{Z})$ :

$$
\mathbf{P}\left\{\frac{\ell^{ \pm}(k, x)}{\sqrt{k}} \geq u_{k}\right\} \leq \mathbf{P}\left\{\frac{\ell(k, x)}{\sqrt{k}} \geq u_{k}\right\} \leq \mathbf{P}\left\{\frac{\ell(k)}{\sqrt{k}} \geq u_{k}\right\} \leq \exp \left(-\frac{u_{k}^{2}}{2}\right)
$$

if $k$ is large enough, $k \geq k_{0}$.

Both the statements and the proofs of the next lemmas about local times are very similar to the ones about BM approximations $\tilde{B}_{m}(t)$ in Lemma 3 .

Lemma 5. For any $C>1$, and for any $K>0$ and $m \geq 1$ such that $K 2^{2 m} \geq N_{4}(C)$, we have

$$
\begin{array}{r}
\mathbf{P}\left\{\sup _{j \in \mathbb{Z}} \sup _{0 \leq t_{k} \leq K}\left|\mathcal{L}_{m+1}\left(t_{k}, x_{j}\right)-\mathcal{L}_{m}\left(t_{k}, x_{j}\right)\right| \geq 6 C K^{\frac{1}{4}}\left(\log _{*} K\right)^{\frac{3}{4}} m^{\frac{3}{4}} 2^{-\frac{m}{2}}\right\} \\
\leq 12\left(K 2^{2 m}\right)^{1-C}
\end{array}
$$

where $t_{k}=k 2^{-2 m}$ and $x_{j}=j 2^{-m}$.

Proof. We are making use of the fact that a RW of length $N=K 2^{2 m}$ typically cannot have values or local times much larger than $\sqrt{N}$. Let us introduce the abbreviations $D_{K, m}=$ 
$C K^{\frac{1}{4}}\left(\log _{*} K\right)^{\frac{3}{4}} m^{\frac{3}{4}} 2^{-\frac{m}{2}}, M=\left(3 C m K \log _{*} K\right)^{\frac{1}{2}} 2^{m}$ (for the "maximum"), $N^{\prime \prime}=\sqrt{3} M$ (for the "maximal" local time), and $K_{m}=\left\lfloor K 2^{2 m}\right\rfloor$. Then using the triangle inequality

$$
\begin{aligned}
& \left|\ell_{m+1}(4 k, 2 j)-2 \ell_{m}(k, j)\right| \\
& \quad \leq\left|\ell_{m+1}\left(T_{m+1}(k), 2 j\right)-2 \ell_{m}(k, j)\right|+\left|\ell_{m+1}(4 k, 2 j)-\ell_{m+1}\left(T_{m+1}(k), 2 j\right)\right| \\
& \quad=: \quad A_{m+1}(k, j)+B_{m+1}(k, j),
\end{aligned}
$$

we get that

$$
\begin{aligned}
\mathbf{P} & \left\{\sup _{j \in \mathbb{Z}} \sup _{0 \leq t_{k} \leq K}\left|\mathcal{L}_{m+1}\left(t_{k}, x_{j}\right)-\mathcal{L}_{m}\left(t_{k}, x_{j}\right)\right| \geq 6 D_{K, m}\right\} \\
& \leq \mathbf{P}\left\{\sup _{|j| \leq M} \sup _{0 \leq t_{k} \leq K} 2^{-m-1}\left|\ell_{m+1}(4 k, 2 j)-2 \ell_{m}(k, j)\right| \geq 6 D_{K, m}\right\} \\
& +\mathbf{P}\left\{\sup _{|j|>M} \sup _{0 \leq t_{k} \leq K}\left|\mathcal{L}_{m+1}\left(t_{k}, x_{j}\right)-\mathcal{L}_{m}\left(t_{k}, x_{j}\right)\right| \geq 6 D_{K, m}\right\} \\
& \leq \sum_{|j| \leq M} \sum_{n=1}^{N^{\prime \prime}} \mathbf{P}\left\{\ell_{m}\left(K_{m}, j\right)=n ; \sup _{0 \leq k \leq K_{m}} 2^{-m-1} A_{m+1}(k, j) \geq 3 D_{K, m}\right\} \\
& +\sum_{|j| \leq M} \sum_{n=N^{\prime \prime}+1}^{K_{m} / 2} \mathbf{P}\left\{\ell_{m}\left(K_{m}, j\right)=n ; \sup _{0 \leq k \leq K_{m}} 2^{-m-1} A_{m+1}(k, j) \geq 3 D_{K, m}\right\} \\
& +\mathbf{P}\left\{\sup _{|j| \leq M} \sup _{0 \leq k \leq K_{m}} 2^{-m-1} B_{m+1}(k, j) \geq 3 D_{K, m}\right\} \\
& +\mathbf{P}\left\{\sup _{|j|>M} \sup _{0 \leq t_{k} \leq K}\left|\mathcal{L}_{m+1}\left(t_{k}, x_{j}\right)-\mathcal{L}_{m}\left(t_{k}, x_{j}\right)\right| \geq 6 D_{K, m}\right\} \\
= & p_{1}+p_{2}+p_{3}+p_{4},
\end{aligned}
$$

We are going to estimate $p_{1}, p_{2}, p_{3}$, and $p_{4}$ one by one.

Estimation of $p_{1}$.

This is the essential part of the proof. Fixing $m \geq 0, j \in[-M, M], n \geq 1$, and given $\ell_{m}\left(K_{m}, j\right)=n$, let $\tau_{0}<\tau_{1}<\cdots<\tau_{n-1}<K_{m}$ denote the random time instants when the "twisted" RW $\tilde{S}_{m}(k)$ is at the point $j$ in the interval $\left[0, K_{m}\right): \tilde{S}_{m}\left(\tau_{i}\right)=j$; then $\tilde{S}_{m+1}\left(T_{m+1}\left(\tau_{i}\right)\right)=2 j$. We define $\tau_{n}=K_{m}$, irrespective whether $\tilde{S}_{m}\left(\tau_{n}\right)=0$ or not. Then we can write that

$$
\begin{aligned}
\ell_{m+1}\left(T_{m+1}\left(K_{m}\right), 2 j\right) & =\sum_{i=1}^{n} \ell_{m+1}\left(T_{m+1}\left(\tau_{i}\right), 2 j\right)-\ell_{m+1}\left(T_{m+1}\left(\tau_{i-1}\right), 2 j\right) \\
& =\sum_{i=1}^{n} \gamma_{n, i} .
\end{aligned}
$$

(The dependence of $\gamma_{n, i}$ on $m$ and $j$ is suppressed in the notation.) Here each random variable $\gamma_{n, i}(i=1, \ldots, n)$ is the number of time instants when $\tilde{S}_{m+1}(k)$ hits the point $2 j$ in the interval $\left[T_{m+1}\left(\tau_{i-1}\right), T_{m+1}\left(\tau_{i}\right)\right)$. This is simply 1 plus the number of the $+1,-1$ or $-1,+1$ pairs of steps of $\tilde{S}_{m+1}(k)$, starting from $T_{m+1}\left(\tau_{i-1}\right)$. Any such sequence ends with a pair $+1,+1$ or $-1,-1$. Clearly, this means that $\left(\gamma_{n, i}\right)_{i=1}^{n}$ is a sequence of independent, geometrically distributed random variables with parameter $p=\frac{1}{2} ; \mathbf{E}\left(\gamma_{n, i}\right)=2$ and $\operatorname{Var}\left(\gamma_{n, i}\right)=2$. 
From the construction of $\tilde{S}_{m+1}(k)$ discussed in Section 2 it is also clear that given $\ell_{m}\left(K_{m}, j\right)=n(n \geq 1)$,

$$
\begin{aligned}
& \sup _{0 \leq k \leq K_{m}}\left|\ell_{m+1}\left(T_{m+1}(k), 2 j\right)-2 \ell_{m}(k, j)\right| \\
& =\sup _{1 \leq i \leq n}\left|\ell_{m+1}\left(T_{m+1}\left(\tau_{i}\right), 2 j\right)-2 \ell_{m}\left(\tau_{i}, j\right)\right|=\sup _{1 \leq r \leq n}\left|\sum_{i=1}^{r} \gamma_{n, i}-2 r\right| .
\end{aligned}
$$

(Note that $\ell_{m}\left(K_{m}, j\right)=0$ implies $\ell_{m+1}\left(T_{m+1}\left(K_{m}\right), 2 j\right)=0$ as well.) Hence

$$
\begin{aligned}
p_{1} & \leq \sum_{|j| \leq M} \sum_{n=1}^{N^{\prime \prime}} \mathbf{P}\left\{\sup _{1 \leq r \leq n}\left|\sum_{i=1}^{r} \gamma_{n, i}-2 r\right| \geq 62^{m} D_{K, m}\right\} \\
& \leq(2 M+1) N^{\prime \prime} \mathbf{P}\left\{\sup _{1 \leq r \leq N^{\prime \prime}}\left|\sum_{i=1}^{r} \frac{\gamma_{i}-2}{\sqrt{2}}\right| \geq 3 \sqrt{2} 2^{m} D_{K, m}\right\}
\end{aligned}
$$

Here we are going to use Lemma 1 with $N^{\prime \prime}$ and $C^{\prime \prime}$. The constant $C^{\prime \prime}$ is chosen so that the error probabilities $p_{1}, p_{2}, p_{3}$, and $p_{4}$ be of the same order: $(2 M+1) N^{\prime \prime}\left(N^{\prime \prime}\right)^{1-C^{\prime \prime}} \leq$ $(2.5 / \sqrt{3})\left(N^{\prime \prime}\right)^{3-C^{\prime \prime}} \approx N^{1-C}$, where $N=K 2^{2 m}$. Then $\left(N^{\prime \prime}\right)^{3-C^{\prime \prime}} \leq\left(K^{\frac{1}{2}} 2^{m}\right)^{3-C^{\prime \prime}}=$ $\left(K 2^{2 m}\right)^{1-C}$ implies $C^{\prime \prime}=2 C+1<3 C$. Since $\log N^{\prime \prime} \leq m \log _{*} C \log _{*} K$ if $K$ or $m$ are large enough, it follows that $\left(2 C^{\prime \prime} N^{\prime \prime} \log N^{\prime \prime}\right)^{\frac{1}{2}} \leq 3 \sqrt{2} 2^{m} D_{K, m}$. Thus we obtain that

$$
p_{1} \leq(5 / \sqrt{3})\left(N^{\prime \prime}\right)^{3-C^{\prime \prime}}<3\left(K 2^{2 m}\right)^{1-C},
$$

if $K 2^{2 m}$ is large enough.

Estimation of $p_{2}$.

Here we are using inequality (11):

$$
\begin{aligned}
p_{2} & \leq \sum_{|j| \leq M} \sum_{n=N^{\prime \prime}+1}^{K_{m} / 2} \mathbf{P}\left\{\ell_{m}\left(K_{m}, j\right)=n\right\} \leq(2 M+1) \mathbf{P}\left\{\ell_{m}\left(K_{m}, 0\right) \geq N^{\prime \prime}\right\} \\
& \leq(2 M+1) \mathbf{P}\left\{\frac{\ell_{m}\left(K_{m}\right)}{\sqrt{K_{m}}} \geq\left(9 C m \log _{*} K\right)^{\frac{1}{2}}\right\} \\
& \leq(2 M+1) \exp \left(-\frac{9}{2} C m \log _{*} K\right) \\
& \leq\left(K 2^{2 m}\right)^{1-C},
\end{aligned}
$$

if $K 2^{2 m}$ is large enough.

Estimation of $p_{3}$.

Here Lemma 2 and inequality (11) will be used. Let $A_{K, m}$ and $N^{\prime}$ be the same as in (5). Then

$$
\begin{aligned}
p_{3} & \leq \mathbf{P}\left\{A_{K, m}\right\} \\
& +\sum_{|j| \leq M} \sum_{k=0}^{K 2^{2 m}} \mathbf{P}\left\{A_{K, m}^{c} ;\left|\ell_{m+1}(4 k, 2 j)-\ell_{m+1}\left(T_{m+1}(k), 2 j\right)\right| \geq 62^{m} D_{K, m}\right\} \\
& \leq 2\left(K 2^{2 m}\right)^{1-C} \\
& +\sum_{|j| \leq M} \sum_{k=0}^{K 2^{2 m}} \mathbf{P}\left\{\sup _{|i-4 k| \leq N^{\prime}}\left|\ell_{m+1}(4 k, 2 j)-\ell_{m+1}(i, 2 j)\right| \geq 62^{m} D_{K, m}\right\} .
\end{aligned}
$$


If $i \geq 4 k$, say, then

$$
\mathbf{P}\left\{\ell_{m+1}(i, 2 j)-\ell_{m+1}(4 k, 2 j) \geq u\right\} \leq \mathbf{P}\left\{\ell_{m+1}(i-4 k, 0) \geq u\right\},
$$

for any $u \geq 0$. For, $\tilde{S}_{m+1}(0)=0$, while $\tilde{S}_{m+1}(4 k)$ can be different from $2 j$ and after the first visit $\tau \geq 4 k$ of $\tilde{S}_{m+1}$ to the point $2 j$ it starts from scratch by the strong Markov property of a simple, symmetric RW. We get that

$$
\begin{aligned}
p_{3} & \leq 2\left(K 2^{2 m}\right)^{1-C}+2(2 M+1) K 2^{2 m} \mathbf{P}\left\{\sup _{0 \leq i-4 k \leq N^{\prime}} \ell_{m+1}(i-4 k) \geq 62^{m} D_{K, m}\right\} \\
& \leq 2\left(K 2^{2 m}\right)^{1-C}+2(2 M+1) K 2^{2 m} \mathbf{P}\left\{\frac{\ell_{m+1}\left(N^{\prime}\right)}{\sqrt{N^{\prime}}} \geq 2^{\frac{1}{4}}(3 C)^{\frac{3}{4}}\left(m \log _{*} K\right)^{\frac{1}{2}}\right\} \\
& \leq 2\left(K 2^{2 m}\right)^{1-C}+2(2 M+1) K 2^{2 m} \exp \left(-2^{-\frac{1}{2}}(3 C)^{\frac{3}{2}} m \log _{*} K\right) \\
& \leq 4\left(K 2^{2 m}\right)^{1-C},
\end{aligned}
$$

if $K 2^{2 m}$ is large enough.

Estimation of $p_{4}$.

Here we are going to apply inequality (4) to $\tilde{S}_{m+1}(4 k)$ and $\tilde{S}_{m}(k)$ :

$$
\begin{aligned}
p_{4} \leq & \mathbf{P}\left\{\sup _{0 \leq t_{k} \leq K}\left|\tilde{B}_{m+1}\left(t_{k}\right)\right| \geq M 2^{-m}\right\}+\mathbf{P}\left\{\sup _{0 \leq t_{k} \leq K}\left|\tilde{B}_{m}\left(t_{k}\right)\right| \geq M 2^{-m}\right\} \\
= & \mathbf{P}\left\{\sup _{0 \leq k \leq K_{m}}\left|\tilde{S}_{m+1}(4 k)\right| \geq 2 M\right\}+\mathbf{P}\left\{\sup _{0 \leq k \leq K_{m}}\left|\tilde{S}_{m}(k)\right| \geq M\right\} \\
\geq & \mathbf{P}\left\{\sup _{0 \leq k \leq K_{m}}\left|\tilde{S}_{m+1}(4 k)\right| \geq\left(2 C K 2^{2(m+1)} \log \left(K 2^{2 m}\right)\right)^{\frac{1}{2}}\right\} \\
& +\mathbf{P}\left\{\sup _{0 \leq k \leq K_{m}}\left|\tilde{S}_{m}(k)\right| \geq\left(2 C K 2^{2 m} \log \left(K 2^{2 m}\right)\right)^{\frac{1}{2}}\right\} \\
\leq & 4\left(K 2^{2 m}\right)^{1-C},
\end{aligned}
$$

if $K 2^{2 m}$ is large enough.

Combining (12), (13), (14), and (15), we get the statement of the lemma.

Lemma 6. For any $C>1$, and for any $K>0$ and $m \geq 1$ such that $K 2^{2 m} \geq N_{5}(C)$, we have

$$
\begin{array}{r}
\mathbf{P}\left\{\sup _{j \in \mathbb{Z}} \sup _{0 \leq t_{k} \leq K}\left|\mathcal{L}_{m+1}^{+}\left(t_{k}, x_{j}\right)-\frac{1}{2} \mathcal{L}_{m}\left(t_{k}, x_{j}\right)\right| \geq 6 C K^{\frac{1}{4}}\left(\log _{*} K\right)^{\frac{3}{4}} m^{\frac{3}{4}} 2^{-\frac{m}{2}}\right\} \\
\leq 12\left(K 2^{2 m}\right)^{1-C},
\end{array}
$$

where $t_{k}=k 2^{-2 m}$ and $x_{j}=j 2^{-m}$. A similar statement holds for $\mathcal{L}_{m+1}^{-}\left(t_{k}, x_{j}\right)$.

Proof. This proof goes similarly as the proof of the previous Lemma 5 , except for the estimation of $p_{1}$, where there are some differences. Hence this is the only point detailed in the sequel. We only discuss the case of $\mathcal{L}_{m+1}^{+}$as the case of $\mathcal{L}_{m+1}^{-}$is analogous.

Fixing $m \geq 0, j \in[-M, M], n \geq 1$, and given $\ell_{m}\left(K_{m}, j\right)=n$, let $\tau_{0}<\tau_{1}<\cdots<$ $\tau_{n-1}<K_{m}$ denote the random time instants when $\tilde{S}_{m}\left(\tau_{i}\right)=j$ in the interval $\left[0, K_{m}\right)$; then 
$\tilde{S}_{m+1}\left(T_{m+1}\left(\tau_{i}\right)\right)=2 j$. We define $\tau_{n}=K_{m}$. Then we can write that

$$
\begin{aligned}
\ell_{m+1}^{+}\left(T_{m+1}\left(K_{m}\right), 2 j\right) & =\sum_{i=1}^{n} \ell_{m+1}^{+}\left(T_{m+1}\left(\tau_{i}\right), 2 j\right)-\ell_{m+1}^{+}\left(T_{m+1}\left(\tau_{i-1}\right), 2 j\right) \\
& =\sum_{i=1}^{n}\left(\alpha_{n, i}+X_{n, i}\right) .
\end{aligned}
$$

(The dependence of $\alpha_{n, i}$ and $X_{n, i}$ on $m$ and $j$ is suppressed in the notation.) Here each random variable $\alpha_{n, i}(i=1, \ldots, n)$ is the number of time instants $k \in\left[T_{m+1}\left(\tau_{i-1}\right), T_{m+1}\left(\tau_{i}\right)\right)$ when $\tilde{S}_{m+1}(k)=2 j, \tilde{S}_{m+1}(k+1)=2 j+1$, and $\tilde{S}_{m+1}(k+2)=2 j$. This is simply the number of the $+1,-1$ pairs of steps of $\tilde{S}_{m+1}(k)$ in the interval $\left[T_{m+1}\left(\tau_{i-1}\right), T_{m+1}\left(\tau_{i}\right)\right)$. Any sequence of $+1,-1$ or $-1,+1$ pairs ends with a pair $+1,+1$ or $-1,-1$. In the former case, $X_{n, i}=1$, in the second case $X_{n, i}=0$. It follows that $\left(\alpha_{n, i}\right)_{i=1}^{n}$ is a sequence of independent, geometrically distributed random variables with parameter $p=\frac{2}{3} ; \mathbf{E}\left(\alpha_{n, i}\right)=\frac{1}{2}$ and $\operatorname{Var}\left(\alpha_{n, i}\right)=\frac{3}{4}$. Further, $\left(X_{n, i}\right)_{i=1}^{n}$ is a sequence of independent indicator variables with parameter $p=\frac{1}{2}$; $\mathbf{E}\left(X_{n, i}\right)=\frac{1}{2}$ and $\operatorname{Var}\left(X_{n, i}\right)=\frac{1}{4}$. The two sequences are also independent.

It is also clear that given $\ell_{m}\left(K_{m}, j\right)=n(n \geq 1)$,

$$
\begin{aligned}
& C_{m+1}(k, j):=\sup _{0 \leq k \leq K_{m}}\left|\ell_{m+1}^{+}\left(T_{m+1}(k), 2 j\right)-\ell_{m}(k, j)\right| \\
& =\sup _{1 \leq i \leq n}\left|\ell_{m+1}^{+}\left(T_{m+1}\left(\tau_{i}\right), 2 j\right)-\ell_{m}\left(\tau_{i}, j\right)\right|=\sup _{1 \leq r \leq n}\left|\sum_{i=1}^{r}\left(\alpha_{n, i}+X_{n, i}\right)-r\right| .
\end{aligned}
$$

Hence in the same way as in the proof of Lemma 5 .

$$
\begin{aligned}
p_{1} & :=\sum_{|j| \leq M} \sum_{n=1}^{N^{\prime \prime}} \mathbf{P}\left\{\ell_{m}\left(K_{m}, j\right)=n ; 2^{-m-1} C_{m+1}(k, j) \geq 3 D_{K, m}\right\} \\
& \leq \sum_{|j| \leq M} \sum_{n=1}^{N^{\prime \prime}} \mathbf{P}\left\{\sup _{1 \leq r \leq n}\left|\sum_{i=1}^{r}\left(\alpha_{n, i}+X_{n, i}\right)-r\right| \geq 62^{m} D_{K, m}\right\} \\
& \leq(2 M+1) N^{\prime \prime} \mathbf{P}\left\{\sup _{1 \leq r \leq N^{\prime \prime}}\left|\sum_{i=1}^{r}\left(\alpha_{i}+X_{n, i}-1\right)\right| \geq 62^{m} D_{K, m}\right\} \\
& <3\left(K 2^{2 m}\right)^{1-C},
\end{aligned}
$$

if $K 2^{2 m}$ is large enough. This ends the proof of the lemma.

Lemma 7. For any $C>1$, and for any $K>0$ and $m \geq 1$ such that $K 2^{2 m} \geq N_{6}(C)$, we have

$$
\begin{array}{r}
\mathbf{P}\left\{\sup _{r \geq 1} \sup _{(t, x) \in[0, K] \times \mathbb{R}}\left|\mathcal{L}_{m+r}(t, x)-\mathcal{L}_{m}(t, x)\right| \geq 79 C K_{*}^{\frac{1}{4}}\left(\log _{*} K\right)^{\frac{3}{4}} m^{\frac{3}{4}} 2^{-\frac{m}{2}}\right\} \\
\leq \frac{15}{1-4^{1-C}}\left(K 2^{2 m}\right)^{1-C},
\end{array}
$$

where $K_{*}=\max \{1, K\}$. 
Proof. STEP 1

$\tilde{B}_{m+1}\left(k 2^{-2(m+1)}\right)$ visits new points $x_{j+\frac{1}{2}}=\left(j+\frac{1}{2}\right) 2^{-m}=(2 j+1) 2^{-m-1}(j \in \mathbb{Z})$ that were not visited by $\tilde{B}_{m}\left(k 2^{-2 m}\right)$. Thus first we need to show an inequality for $\mathcal{L}_{m+1}\left(t_{k}, x_{j+\frac{1}{2}}\right)$, similar to the one in Lemma 5

$$
\begin{array}{r}
\mathbf{P}\left\{\sup _{j \in \mathbb{Z}} \sup _{0 \leq t_{k} \leq K}\left|\mathcal{L}_{m+1}\left(t_{k}, x_{j+\frac{1}{2}}\right)-\mathcal{L}_{m}\left(t_{k}, x_{j+\frac{1}{2}}\right)\right| \geq 9 C K^{\frac{1}{4}}\left(\log _{*} K\right)^{\frac{3}{4}} m^{\frac{3}{4}} 2^{-\frac{m}{2}}\right\} \\
\leq 15\left(K 2^{2 m}\right)^{1-C} .
\end{array}
$$

Since $\mathcal{L}_{m}\left(t_{k}, x_{j+\frac{1}{2}}\right)$ is obtained by linear interpolation and

$$
\ell_{m+1}\left(T_{m+1}(k), 2 j+1\right)=\ell_{m+1}^{+}\left(T_{m+1}(k), 2 j\right)+\ell_{m+1}^{-}\left(T_{m+1}(k), 2 j+2\right)
$$

(note that $\tilde{S}_{m+1}\left(T_{m+1}(k)\right)$ never equals $2 j+1$ ), it follows that

$$
\begin{aligned}
& \left|\mathcal{L}_{m+1}\left(t_{k}, x_{j+\frac{1}{2}}\right)-\mathcal{L}_{m}\left(t_{k}, x_{j+\frac{1}{2}}\right)\right| \\
& \quad=2^{-m-1}\left|\ell_{m+1}(4 k, 2 j+1)-\ell_{m}(k, j)-\ell_{m}(k, j+1)\right| \\
& \quad \leq 2^{-m-1}\left|\ell_{m+1}\left(T_{m+1}(k), 2 j+1\right)-\ell_{m}(k, j)-\ell_{m}(k, j+1)\right| \\
& \quad+2^{-m-1}\left|\ell_{m+1}(4 k, 2 j+1)-\ell_{m+1}\left(T_{m+1}(k), 2 j+1\right)\right| \\
& \quad \leq 2^{-m-1}\left|\ell_{m+1}^{+}\left(T_{m+1}(k), 2 j\right)-\ell_{m}(k, j)\right| \\
& \quad+2^{-m-1}\left|\ell_{m+1}^{-}\left(T_{m+1}(k), 2 j+2\right)-\ell_{m}(k, j+1)\right| \\
& \quad+\quad 2^{-m-1}\left|\ell_{m+1}(4 k, 2 j+1)-\ell_{m+1}\left(T_{m+1}(k), 2 j+1\right)\right| \\
& \quad=: F_{m+1}(k, j)+G_{m+1}(k, j)+H_{m+1}(k, j) .
\end{aligned}
$$

From this point the proof of (17) goes similarly as the proof of Lemma 5 , except for the estimation of $p_{1}$, where there are some differences. Hence this is the only point detailed here. By (16),

$$
\begin{aligned}
p_{1} & :=\sum_{|j| \leq M} \sum_{n=1}^{N^{\prime \prime}} \mathbf{P}\left\{\ell_{m}\left(K_{m}, j\right)=n ; \sup _{0 \leq k \leq K_{m}} F_{m+1}(k, j) \geq 3 D_{K, m}\right\} \\
& +\sum_{|j| \leq M} \sum_{n=1}^{N^{\prime \prime}} \mathbf{P}\left\{\ell_{m}\left(K_{m}, j+1\right)=n ; \sup _{0 \leq k \leq K_{m}} G_{m+1}(k, j) \geq 3 D_{K, m}\right\} \\
& <6\left(K 2^{2 m}\right)^{1-C},
\end{aligned}
$$

if $K 2^{2 m}$ is large enough.

STEP 2

Let $D_{K, m}^{*}=C K_{*}^{\frac{1}{4}}\left(\log _{*} K\right)^{\frac{3}{4}} m^{\frac{3}{4}} 2^{-\frac{m}{2}}$. By Lemma [5,

$$
\sup _{j \in \mathbb{Z}} \sup _{0 \leq t_{k} \leq K}\left|\mathcal{L}_{m+1}\left(t_{k}, x_{j}\right)-\mathcal{L}_{m}\left(t_{k}, x_{j}\right)\right|<6 D_{K, m}^{*},
$$

except for an event of probability not exceeding $12\left(K 2^{2 m}\right)^{1-C}$. Also, by (17),

$$
\sup _{j \in \mathbb{Z}} \sup _{0 \leq t_{k} \leq K}\left|\mathcal{L}_{m+1}\left(t_{k}, x_{j+\frac{1}{2}}\right)-\mathcal{L}_{m}\left(t_{k}, x_{j+\frac{1}{2}}\right)\right|<9 D_{K, m}^{*},
$$

except for an event of probability not exceeding $15\left(K 2^{2 m}\right)^{1-C}$. 
Consider an interval $\left[t_{k}, t_{k+1}\right]$. Clearly, $0 \leq \mathcal{L}_{m}\left(t_{k+1}, x_{j}\right)-\mathcal{L}_{m}\left(t_{k}, x_{j}\right) \leq 2^{-m} \leq 2^{-\frac{1}{2}} D_{K, m}^{*}$. On the other hand, $\tilde{B}_{m+1}$ makes 4 steps on this interval. Thus $0 \leq \mathcal{L}_{m+1}\left(t_{k+1}, x_{j}\right)-$ $\mathcal{L}_{m+1}\left(t_{k}, x_{j}\right) \leq 2 \cdot 2^{-m-1}=2^{-m}$ and $0 \leq \mathcal{L}_{m+1}\left(t_{k+1}, x_{j+\frac{1}{2}}\right)-\mathcal{L}_{m+1}\left(t_{k}, x_{j+\frac{1}{2}}\right) \leq 2^{-m}$ as well. Since $\mathcal{L}_{m}(t, x)$ and $\mathcal{L}_{m+1}(t, x)$ are obtained by linear interpolation for real $t$ and $x$, it follows that

$$
\mathbf{P}\left\{\sup _{(t, x) \in[0, K] \times \mathbb{R}}\left|\mathcal{L}_{m+1}(t, x)-\mathcal{L}_{m}(t, x)\right| \geq\left(9+2^{-\frac{1}{2}}\right) D_{K, m}^{*}\right\} \leq 15\left(K 2^{2 m}\right)^{1-C} .
$$

From this point the proof is the same as the last part of the proof of Lemma 3(b), except for the constant multipliers. Since $\frac{65}{8}\left(9+2^{-\frac{1}{2}}\right)<79$, this proves the statement of the lemma.

By the Borel-Cantelli lemma, the previous Lemma 7 leads to an alternative definition of the local time of Brownian motion $W$ obtained in Theorem 1 , via a sequence of continuous local times of the approximations $\tilde{B}_{m}$.

Theorem 3. On any strip $[0, K] \times \mathbb{R}$ the sequence $\left(\mathcal{L}_{m}(t, x)\right)$ almost surely uniformly converges as $m \rightarrow \infty$ and the limit is a process $\mathcal{L}(t, x)$ jointly continuous in $(t, x)$, the local time of Brownian motion $W(t)$. For any $C>1$, and for any $K>0$ and $m \geq 1$ such that $K 2^{2 m} \geq N_{6}(C)$, we have

$$
\begin{aligned}
\mathbf{P} & \left\{\sup _{(t, x) \in[0, K] \times \mathbb{R}}\left|\mathcal{L}(t, x)-\mathcal{L}_{m}(t, x)\right| \geq 79 C K_{*}^{\frac{1}{4}}\left(\log _{*} K\right)^{\frac{3}{4}} m^{\frac{3}{4}} 2^{-\frac{m}{2}}\right\} \\
& \leq \frac{15}{1-4^{1-C}}\left(K 2^{2 m}\right)^{1-C} .
\end{aligned}
$$

Now using the Borel-Cantelli lemma we get that for any fixed $K>0$ there is a constant $c_{K}=80 K_{*}^{\frac{1}{4}}\left(\log _{*} K\right)^{\frac{3}{4}}$ (taking $C=1+\frac{1}{79}$, say) such that almost surely,

$$
\limsup _{m \rightarrow \infty} m^{-\frac{3}{4}} 2^{\frac{m}{2}} \sup _{(t, x) \in[0, K] \times \mathbb{R}}\left|\mathcal{L}(t, x)-\mathcal{L}_{m}(t, x)\right|<c_{K} .
$$

Also, for any fixed $m \geq 1$ there is a constant $c_{m}=159 m^{\frac{3}{4}} 2^{-\frac{m}{2}}$ (taking $C=2+\frac{1}{79}$, say) such that almost surely,

$$
\limsup _{K \rightarrow \infty} K^{-\frac{1}{4}}(\log K)^{-\frac{3}{4}} \sup _{(t, x) \in[0, K] \times \mathbb{R}}\left|\mathcal{L}(t, x)-\mathcal{L}_{m}(t, x)\right|<c_{m} .
$$

This also follows by the Borel-Cantelli lemma, in a similar way as (7) did.

One has similar convergence results for the one-sided local times as well.

Theorem 4. On any strip $[0, K] \times \mathbb{R}$ the sequence $\left(\mathcal{L}_{m}^{+}(t, x)\right)$ almost surely uniformly converges as $m \rightarrow \infty$ to the one half of the Brownian local time $\mathcal{L}(t, x)$. For any $C>1$, and for any $K>0$ and $m \geq 1$ such that $K 2^{2 m} \geq N_{7}(C)$, we have

$$
\begin{aligned}
\mathbf{P} & \left\{\sup _{(t, x) \in[0, K] \times \mathbb{R}}\left|\frac{1}{2} \mathcal{L}(t, x)-\mathcal{L}_{m+1}^{+}(t, x)\right| \geq 50 C K_{*}^{\frac{1}{4}}\left(\log _{*} K\right)^{\frac{3}{4}} m^{\frac{3}{4}} 2^{-\frac{m}{2}}\right\} \\
& \leq \frac{30}{1-4^{1-C}}\left(K 2^{2 m}\right)^{1-C} .
\end{aligned}
$$

Similar statements hold for $\left(\mathcal{L}_{m+1}^{-}(t, x)\right)$ as well. 
Proof. STEP 1

First we need to show an inequality similar to (17) for points $x_{j+\frac{1}{2}}$ :

$$
\begin{aligned}
\mathbf{P} & \left\{\sup _{j \in \mathbb{Z}} \sup _{0 \leq t_{k} \leq K}\left|\mathcal{L}_{m+1}^{+}\left(t_{k}, x_{j+\frac{1}{2}}\right)-\frac{1}{2} \mathcal{L}_{m}\left(t_{k}, x_{j+\frac{1}{2}}\right)\right| \geq 9 C K^{\frac{1}{4}}\left(\log _{*} K\right)^{\frac{3}{4}} m^{\frac{3}{4}} 2^{-\frac{m}{2}}\right\} \\
& \leq 15\left(K 2^{2 m}\right)^{1-C} .
\end{aligned}
$$

Our argument will follow a similar path to the ones in Lemmas 6 and 7 Since $\mathcal{L}_{m}\left(t_{k}, x_{j+\frac{1}{2}}\right)$ is obtained by linear interpolation, it follows that

$$
\begin{aligned}
& \left|\mathcal{L}_{m+1}^{+}\left(t_{k}, x_{j+\frac{1}{2}}\right)-\frac{1}{2} \mathcal{L}_{m}\left(t_{k}, x_{j+\frac{1}{2}}\right)\right| \\
& \quad=2^{-m-1}\left|\ell_{m+1}^{+}(4 k, 2 j+1)-\frac{1}{2}\left(\ell_{m}(k, j)+\ell_{m}(k, j+1)\right)\right| \\
& \quad \leq \quad 2^{-m-1}\left|\ell_{m+1}^{+}\left(T_{m+1}(k), 2 j+1\right)-\frac{1}{2}\left(\ell_{m}(k, j)+\ell_{m}(k, j+1)\right)\right| \\
& \quad+\quad 2^{-m-1}\left|\ell_{m+1}^{+}(4 k, 2 j+1)-\ell_{m+1}^{+}\left(T_{m+1}(k), 2 j+1\right)\right| \\
& \quad \leq \quad 2^{-m-1}\left|\ell_{m+1}^{++}\left(T_{m+1}(k), 2 j+1\right)-\frac{1}{2} \ell_{m}(k, j)\right| \\
& \quad+\quad 2^{-m-1}\left|\ell_{m+1}^{-+}\left(T_{m+1}(k), 2 j+1\right)-\frac{1}{2} \ell_{m}(k, j+1)\right| \\
& \quad+\quad 2^{-m-1}\left|\ell_{m+1}^{+}(4 k, 2 j+1)-\ell_{m+1}^{+}\left(T_{m+1}(k), 2 j+1\right)\right|
\end{aligned}
$$

where we applied the notations for up-crossing and up-bouncing local times, introduced above.

Here, analogously to the proof of Lemma 6, given $\ell_{m}\left(K_{m}, j\right)=n$, one has

$$
\ell_{m+1}^{++}\left(T_{m+1}\left(K_{m}\right), 2 j+1\right)=\sum_{i=1}^{n} X_{n, i}
$$

where $X_{n, i}=1$ if a sequence of $+1,-1$ or $-1,+1$ pairs of steps of $\tilde{S}_{m+1}(k)$ in the interval $\left[T_{m+1}\left(\tau_{i-1}\right), T_{m+1}\left(\tau_{i}\right)\right)$ ends with a pair $+1,+1$ and 0 otherwise; $\tau_{0}<\tau_{1}<\cdots<\tau_{n-1}<K_{m}$ are the random time instants when $\tilde{S}_{m}\left(\tau_{i}\right)=j$ in the interval $\left[0, K_{m}\right)$ and $\tau_{n}=K_{m}$. Then $\left(X_{n, i}\right)_{i=1}^{n}$ is a sequence of independent indicator variables with parameter $p=\frac{1}{2}$; $\mathbf{E}\left(X_{n, i}\right)=\frac{1}{2}$ and $\operatorname{Var}\left(X_{n, i}\right)=\frac{1}{4}$. Further, given $\ell_{m}\left(K_{m}, j\right)=n$,

$$
\sup _{0 \leq k \leq K_{m}}\left|\ell_{m+1}^{++}\left(T_{m+1}(k), 2 j+1\right)-\frac{1}{2} \ell_{m}(k, j)\right|=\sup _{1 \leq r \leq n}\left|\sum_{i=1}^{r}\left(X_{n, i}-\frac{1}{2}\right)\right| .
$$

Similarly, given $\ell_{m}\left(K_{m}, j+1\right)=n$,

$$
\ell_{m+1}^{-+}\left(T_{m+1}\left(K_{m}\right), 2 j+1\right)=\sum_{i=1}^{n} \beta_{n, i}
$$

where $\beta_{n, i}$ is simply the number of the $-1,+1$ pairs of steps of $\tilde{S}_{m+1}(k)$ in the interval $\left[T_{m+1}\left(\tau_{i-1}\right), T_{m+1}\left(\tau_{i}\right)\right) ; \tau_{0}<\tau_{1}<\cdots<\tau_{n-1}<K_{m}$ are the random time instants when $\tilde{S}_{m}\left(\tau_{i}\right)=j+1$ in the interval $\left[0, K_{m}\right)$ and $\tau_{n}=K_{m}$. It follows that $\left(\beta_{n, i}\right)_{i=1}^{n}$ is a sequence of 
independent, geometrically distributed random variables with parameter $p=\frac{2}{3} ; \mathbf{E}\left(\beta_{n, i}\right)=\frac{1}{2}$ and $\operatorname{Var}\left(\beta_{n, i}\right)=\frac{3}{4}$. Moreover, given $\ell_{m}\left(K_{m}, j+1\right)=n$,

$$
\sup _{0 \leq k \leq K_{m}}\left|\ell_{m+1}^{-+}\left(T_{m+1}(k), 2 j+1\right)-\frac{1}{2} \ell_{m}(k, j+1)\right|=\sup _{1 \leq r \leq n}\left|\sum_{i=1}^{r}\left(\beta_{n, i}-\frac{1}{2}\right)\right| .
$$

From this point the proof of (21) is essentially the same as the proof of (17).

STEP 2

By Lemma 6 and formula (21), using the same argument as in STEP 2 of Lemma 7 , it follows that

$$
\mathbf{P}\left\{\sup _{(t, x) \in[0, K] \times \mathbb{R}}\left|\mathcal{L}_{m+1}^{+}(t, x)-\frac{1}{2} \mathcal{L}_{m}(t, x)\right| \geq\left(9+2^{-\frac{1}{2}}\right) D_{K, m}^{*}\right\} \leq 15\left(K 2^{2 m}\right)^{1-C} .
$$

Since $\left|\frac{1}{2} \mathcal{L}(t, x)-\mathcal{L}_{m+1}^{+}(t, x)\right| \leq\left|\frac{1}{2} \mathcal{L}(t, x)-\frac{1}{2} \mathcal{L}_{m}(t, x)\right|+\left|\frac{1}{2} \mathcal{L}_{m}(t, x)-\mathcal{L}_{m+1}^{+}(t, x)\right|$, formula (22) and Theorem 3 give that

$$
\begin{aligned}
\mathbf{P} & \left\{\sup _{(t, x) \in[0, K] \times \mathbb{R}}\left|\frac{1}{2} \mathcal{L}(t, x)-\mathcal{L}_{m+1}^{+}(t, x)\right| \geq\left(\frac{79}{2}+9+2^{-\frac{1}{2}}\right) D_{K, m}^{*}\right\} \\
& \leq\left(\frac{15}{1-4^{1-C}}+15\right)\left(K 2^{2 m}\right)^{1-C} .
\end{aligned}
$$

This proves the theorem.

We mention that similar convergence results can be shown for up- and down-crossing or up- and down-bouncing local times as well.

\section{References}

[1] Bass, R.F. and Khoshnevisan, D. (1993) Rates of convergence to Brownian local time. Stochastic Process. Appl. 47 197-213.

[2] Borodin, A.N. (1989) Brownian local time. Russian Math. Surveys 44, 1-51.

[3] Csáki, E. and Révész, P. (1983) Strong invariance for local times. Z. Wahrscheinlichkeitstheorie und Verw. Gebiete 62, 263-278.

[4] Csörgő, M. and Horváth, L. (1989) On best possible approximations of local time. Statist. Probab. Lett. 8 301-306.

[5] Kiefer, J. (1969) On the deviation in the Skorokhod-Strassen approximation scheme. Z. Wahrscheinlichkeitstheorie und Verw. Gebiete 13 321-332.

[6] Knight, F.B. (1962) On the random walk and Brownian motion. Trans. Amer. Math. Soc. 103, 218-228.

[7] Knight, F.B. (1963) Random walks and a sojourn density process of Brownian motion. Trans. Amer. Math. Soc. 107, 56-86.

[8] Révész, P. (1981) Local times and invariance. In: Analytic Methods in Probability Theory. Lecture Notes in Math. No. 861. pp. 128-145. Springer, Berlin. 
[9] Révész, P. (1990) Random Walk in Random and Non-Random Environments. World Scientific, Singapore.

[10] Szabados, T. (1996) An elementary introduction to the Wiener process and stochastic integrals. Studia Sci. Math. Hung. 31, 249-297.

[11] Szabados, T. (2001) Strong approximation of fractional Brownian motion by moving averages of simple random walks. Stochastic Process. Appl. 92, 31-60.

[12] Székely, B. and Szabados, T. (2004) Strong approximation of continuous local martingales by simple random walks. Studia Sci. Math. Hung. 41, 101-126.

[13] Trotter, H. F. (1958) A property of Brownian motion paths. Illinois J. Math. 2, 425-433. 